\title{
Plantar Soft-tissue Stress states in standing: a Three-Dimensional Finite Element Foot Modeling Study
}

\author{
Wen-Ming, Chen - Peter Vee-Sin, Lee - Tae-Yong, Lee \\ (Division of Bioengineering, National University of Singapore, Singapore)
}

\begin{abstract}
W. M. CHEN, V. S. LEE, and T. Y. LEE, Plantar Soft-tissue Stress states in standing: a Three-Dimensional Finite Element Foot Modeling Study. Korean Journal of Sport Biomechanics, Vol. 19, No. 2, pp. 197-204, 2009. It has been hypothesized that foot ulceration might be internally initiated. Current instruments which merely allow superficial estimate of plantar loading acting on the foot, severely limit the scope of many biomechanical/clinical studies on this issue. Recent studies have suggested that peak plantar pressure may be only $65 \%$ specific for the development of ulceration. These limitations are at least partially due to surface pressures not being representative of the complex mechanical stress developed inside the subcutaneous plantar soft-tissue, which are potentially more relevant for tissue breakdown. This study established a three-dimensional and nonlinear finite element model of a human foot complex with comprehensive skeletal and soft-tissue components capable of predicting both the external and internal stresses and deformations of the foot. The model was validated by experimental data of subject-specific plantar foot pressure measures. The stress analysis indicated the internal stresses doses were site-dependent and the observation found a change between 1.5 to 4.5 times the external stresses on the foot plantar surface. The results yielded insights into the internal loading conditions of the plantar soft-tissue, which is important in enhancing our knowledge on the causes of foot ulceration and related stress-induced tissue breakdown in diabetic foot.
\end{abstract}

KEYWORDS : FOOT PLANTAR SOFT-TISSUE, PLANTAR ULCER, TISSUE STRESS STATES, FINITE ELEMENT MODEL

*Corresponding Author : Tae-Yong Lee

Division of Bioengineering, National University of Singapore, Singapore.

Tel : +65-6516-1471 / Fax : +65-6872-3069

E-mail : bielt@nus,edu.sg 


\section{I . Introduction}

It has been realized for many years that diabetics are prone to ulceration and gangrene of the feet. Foot ulcers are among the most devastating complications of diabetes (Boulton et al., 2005). The lifetime risk for a patient with diabetes developing a foot ulcer could be as high as $25 \%$, and limb amputation, the most costly and feared consequence of a foot ulcer, occurs 10 to 30 times more often in diabetic patients than in the general population (Singh et al., 2005). Although the etiology of neuropathic diabetic foot ulcers is still being investigated, the major contributing factors are believed to be the presence of LOPS due to peripheral neuropathy and repetitive excessive mechanical stresses acting on the sole of the insensate foot during gait, such as walking.In the past, many in vivo and in vitro studies that addressed foot risks have focused on delineating the potential overloading of the foot by plantar pressure measurements (Duckworth et al., 1985, Alexander et al., 1990). However, given a combination of complex geometry and nonlinear material behavior of the subcutaneous plantar soft-tissue, the force acting on the plantar surface may lead to highly inhomogeneous internal mechanical loading conditions. This is especially true for the sites underneath the prominence of the metatarsal heads (MTH), in which stress concentration is caused by the contact between soft-tissue and irregular-shaped bony structures (Mueller et al., 2006, Morag \& Cavanagh, 1999). Therefore, the relationship between the plantar pressure and internal stress is unlikely to be simple. High-resolution magnetic resonance imaging (HR-MRI) has revealed the disruption of the subcutaneous plantar tissue in diabetic feet with micro-haemorrhage (Brash et al., 1996). If it is true that ulceration of the plantar soft-tissue results from excessive mechanical stress, then it appears reasonable that foot ulceration may be internally initiated and work upwards to the outermost skin level.

It is not surprising that there are significant limitations in using a single critical level of surface plantar foot pressure to identify patients at risk for neuropathic foot ulceration (Masson et al., 1989, Armstrong et al., 1998). Recent studies have suggested that peak plantar pressure may only be $65 \%$ specific for the development of ulcers (Cavanagh et al., 2000). These limitations are at least partially due to surface pressures not being representative of the complex mechanical stresses that developed inside the subcutaneous plantar soft-tissue, which are more likely to be the cause of tissue breakdown.

The finite element method (FEM) is a powerful mathematical tool which allows internal stress and strain analyses of complex structures with geometrical and material nonlinearity. The loading on the plantar soft-tissue of the foot is represented by a complex interplay of the foot skeleton, cartilages, muscles, ligaments, fascia, and the external environment. Although in-vivo plantar soft tissue deformation measurements using imaging techniques like sonography and radiography have been reported in the literature, FE models verified through such data are still lacking. Such models can increase the confidence of FE models and exploit the models' main advantage to predict the foot's internal stresses and deformations.

Therefore, the aim of this study is to develop a detailed three-dimensional finite element model of the foot validated by subject specific plantar foot pressure measurements. Following, the feasibility of the model to predict the internal plantar soft tissue deformation and stress will be evaluated.

\section{Methods}

\section{Foot Plantar Pressure measurement}


Table 1. Summary of FE model listing element type and material properties for different model entities.

\begin{tabular}{|c|c|c|c|c|}
\hline Entity & Element type & $\mathrm{E}(\mathrm{MPa}), \mathrm{v}$ & Cross-section & Reference \\
\hline Bone & $\begin{array}{l}\text { 4-node tetrahedral } \\
\text { continuum }\end{array}$ & $7300,0.3$ & - & $\begin{array}{l}\text { Cortical and cancellous bone properties weighted by } \\
\text { Nakamura et al (Nakamura et al., 1981) }\end{array}$ \\
\hline Cartilage & $\begin{array}{l}\text { 4-node tetrahedral } \\
\text { continuum }\end{array}$ & $1.01,0.4$ & - & Athanasiou et al., (Athanasiou et al., 1998) \\
\hline Ligament & $\begin{array}{l}\text { 2-node tension-only } \\
\text { truss }\end{array}$ & $260,0.4$ & 18.4 & Siegler et al., (Siegler et al., 1988) \\
\hline $\begin{array}{l}\text { Plantar } \\
\text { fascia }\end{array}$ & $\begin{array}{l}\text { 2-node tension-only } \\
\text { truss }\end{array}$ & $350,0.4$ & 290.7 & (Wright and Rennels, 1964)) \\
\hline $\begin{array}{l}\text { Plantar } \\
\text { soft-tissue }\end{array}$ & $\begin{array}{l}\text { 4-node tetrahedral } \\
\text { continuum }\end{array}$ & Hyperelastic & - & $\begin{array}{l}\text { Stress-strain curve determined by Lemmon et } \\
\text { al., (Lemmon et al., 1997) }\end{array}$ \\
\hline Ground & $\begin{array}{l}\text { 8-node hexahedral } \\
\text { continuum }\end{array}$ & $72000,0.33$ & - & Aluminum-Alloy Standard 2014-T6 \\
\hline
\end{tabular}

Table 2. Parameters computed by ABAQUS forthe incompressible hyperelastic material model in polynomial form to characterize plantar soft-tissue behavior.

\begin{tabular}{|c|c|c|c|}
\hline The polynomial form of the strain energy potential & \multicolumn{2}{|l|}{ Parameters } & Value \\
\hline \multirow{8}{*}{$\begin{array}{l}U=\sum_{i+j=1}^{N} C_{i j}\left(\bar{I}_{1}-3\right)^{i}\left(\bar{I}_{2}-3\right)^{j}+\sum_{i=1}^{N} \frac{1}{D_{i}}\left(J_{e l}-1\right)^{2 i} \\
\text { where } \mathrm{U} \text { is the strain energy potential; Jel is the elastic } \\
\text { volume ratio; } \bar{I}_{1} \text { and } \bar{I}_{2} \text { are measurements of the } \\
\text { distortion in the material; and } \mathrm{N}, \mathrm{Cij} \text { and } \mathrm{Di} \text { are material } \\
\text { parameters. }\end{array}$} & Order & $\mathrm{N}$ & 2 \\
\hline & \multirow{5}{*}{$\begin{array}{l}\text { Gij describes the } \\
\text { shear behavior of the material } \\
(\mathrm{Nmm}-2)\end{array}$} & $\mathrm{C} 10$ & 0.08556 \\
\hline & & $\mathrm{CO}$ & -0.05841 \\
\hline & & $\mathrm{C} 20$ & 0.03900 \\
\hline & & C11 & -0.02319 \\
\hline & & $\mathrm{CO} 2$ & 0.00851 \\
\hline & \multirow{2}{*}{$\begin{array}{l}\text { Di introduces compressibility } \\
\text { (mm2N-1) }\end{array}$} & D1 & 3.65273 \\
\hline & & $\mathrm{D} 2$ & 0.00000 \\
\hline
\end{tabular}

A barefoot plantar pressure foot pressure test was conducted using the F-Scan In-shoe Pressure System (Software v. 5.03, Tekscan. Boston, MA) on a 26 year-old male subject who was free of any foot abnormalities and pathologies. The pressure sensors have a high spatial resolution (4 sensing elements per $\mathrm{cm} 2)$ and are ultra-thin $(0.15 \mathrm{~mm})$, thus the interfacial pressure between the foot plantar and the ground can be captured effectively with minimal intrusion. The subject was instructed to stand barefoot on a metal plate (Aluminum-Alloy Standard 2014-T6) which represents the ground. As the material property of the plate (the ground) is known, it can be defined in the FE model (density $=2800 \mathrm{~kg} / \mathrm{m} 3$, Young's modulus $=$
$72 \mathrm{GPa}$, Poisson's ratio $=0.33$ ). The pressure sensor was inserted between the foot plantar and the ground. In order to produce reliable measurements, five static plantar pressure trials were obtained from the subject's right foot. In each test, plantar pressure distribution (force pattern), peak plantar pressure and center of pressure were recorded.

\section{Finite Element Modeling}

The 3-D geometry of the soft tissue-skeletal foot and an-kle joint complex was created based on the coronal com-puter tomography (CT) scan of the right foot of a male adult who was free of any foot 
abnormalities and pathologies. Foot structures such as articular cartilages, ligaments and plantar fascia that could not be reconstructed from $\mathrm{CT}$ were determined through magnetic resonance (MR) imaging and histological observations. 30 bony parts with articular carti-lages, including sesamoids, were created individually and then were enveloped into a mass of foot soft tissue in which the muscular constitutions were not separately identified. A total of 5 sub-bands of plantar fascia and 134 major liga-ments (Ligs) were also incorporated into the model to pas-sively stabilize the cartilage-mediated bony joints: 14 ankle Ligs, 11 talocalcaneal Ligs, 14 talonavicular Ligs, 9 cal-caneocuboidal Ligs, 13 navicocuneiform Ligs, 27 tar-sometatarsal Ligs, and other 46 short Ligs.

In order to simulate the joint interactions among the tibia, fibula, calcaneous, talus, navicular, cuboid, cuneiforms, and metatarsals, ABAQUS sliding "contact" conditions were defined accordingly, which allow bones to slide frictionless over one another. Since the joint surfaces were all wrapped with articular cartilages, the governing stiffness during contact was set to be 1.01MPa which is consistent with the compressive material properties of the foot joint cartilage (Athanasiou et al., 1998). Both the bony structures and soft-tissue were meshed with 4-noded tetrahedral elements, while the ligaments and plantar fascia were represented by 2-noded tension-only truss elements. A second-order polynomial hyperelastic material model was applied to incorporate the nonlinear properties into the pad soft tissue. Other structures were assumed to behavior in linear isotropic manners.

During static standing, an estimated ground reaction force of $325 \mathrm{~N}$ (half of the $\mathrm{BW}$ ) was applied to the foot model through uniformly distributed pressure. An estimated gastrocnemius-soleus muscle force of $162 \mathrm{~N}$ acting through Achilles tendon to the calcaneus was also applied by using force vectors, and the superior part of tibia, fibula and soft tissue were all fixed by all degree of freedom. In addition, a frictional contact interaction was established between the foot plantar surface and the ground.

\section{Validation}

The FE computed axial compressive stress on the ground (along Y-direction, perpendicular to the contacting surface of foot plantar) were compared to the plantar pres-sure distribution measured using F-scan. Quantitative re-sults on nine clinically significant regions as defined by rectangular regions of interest (ROI) on plantar soft-tissue were chosen for comparison: the subhallucal (H), the 1st submetatarsal [medial M1(M) and lateral M1(L) sesamoids], the 2nd to 5th submetatarsal (M2 to M5), the lateral submidfoot $(\mathrm{L})$ and the subcalcaneal $(\mathrm{C})$ region. Moreover, the FE computed center of contact pressure (COP), peak contact stress (PCS), and total contact area (TCA) were also compared with the experimental measures.

\section{Regional Tissue stress analysis}

The internal stress distribution was examined within the boundary of each of the nine ROI. Peak stress level calculated from the innermost plantar soft-tissue which interfaces with the foot bones represents the peak internal stress. It was expressed in terms of von Mises stress (v), which has previously been used as a parameter related to the strength of biological tissues of bone (Keyak et al., 1998) and plantar soft-tissue (Gefen, 2003). It provided a measure of shear linked by the von Mises yield criterion which is based on three principal normal stresses ( $x x, y y$ and $z z)$, in the form of:

$$
\sigma_{v}=\sqrt{2} / 2\left[\left(\sigma_{x x}-\sigma_{y y}\right)^{2}+\left(\sigma_{y y}-\sigma_{z z}\right)^{2}+\left(\sigma_{z z}-\sigma_{x x}\right)^{2}\right]^{1 / 2}(1)
$$




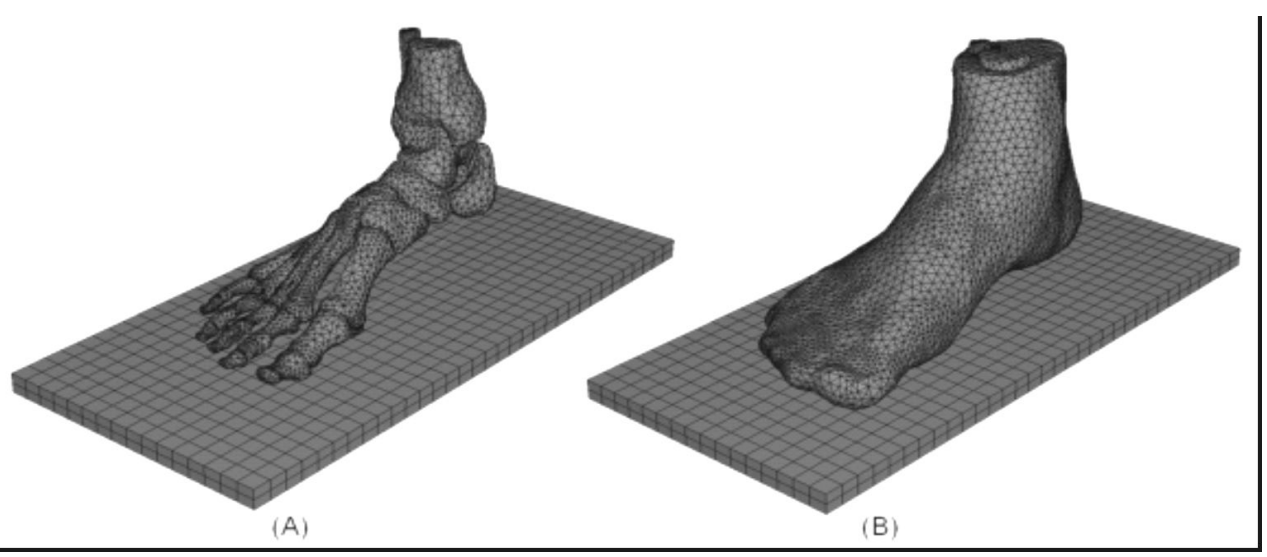

Figure 1. FE model of the skeletal structures of the human foot inclusive of the ligaments and fascia (A), enveloped by a homogenous mass of soft-tissue under the skin (B), standing on the metal plate (C) i.e. ground

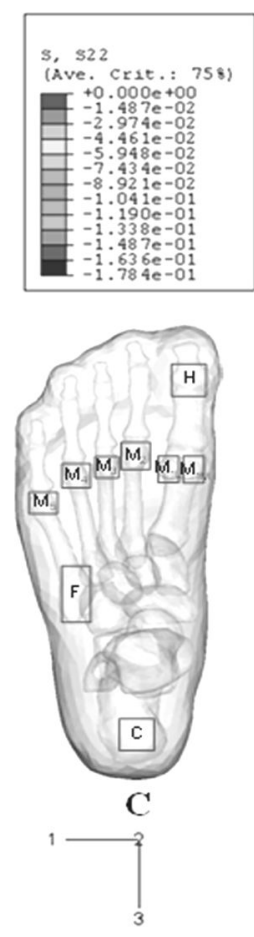

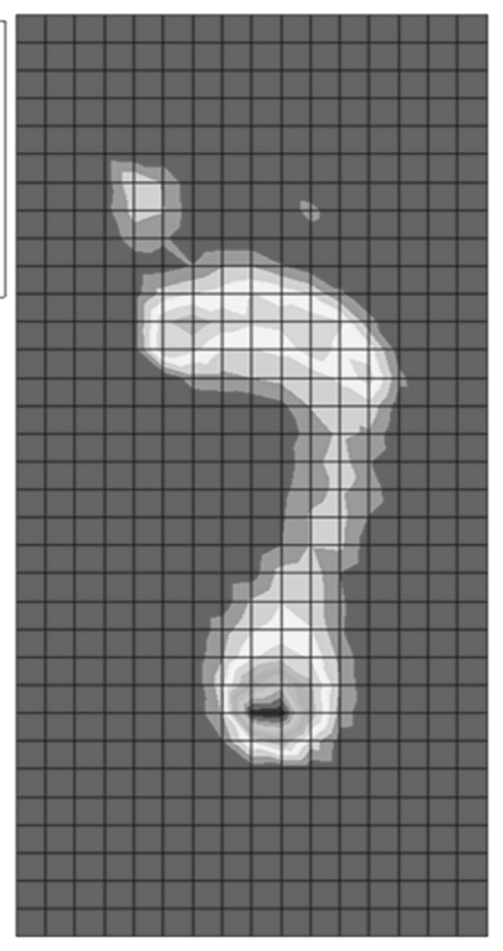

A

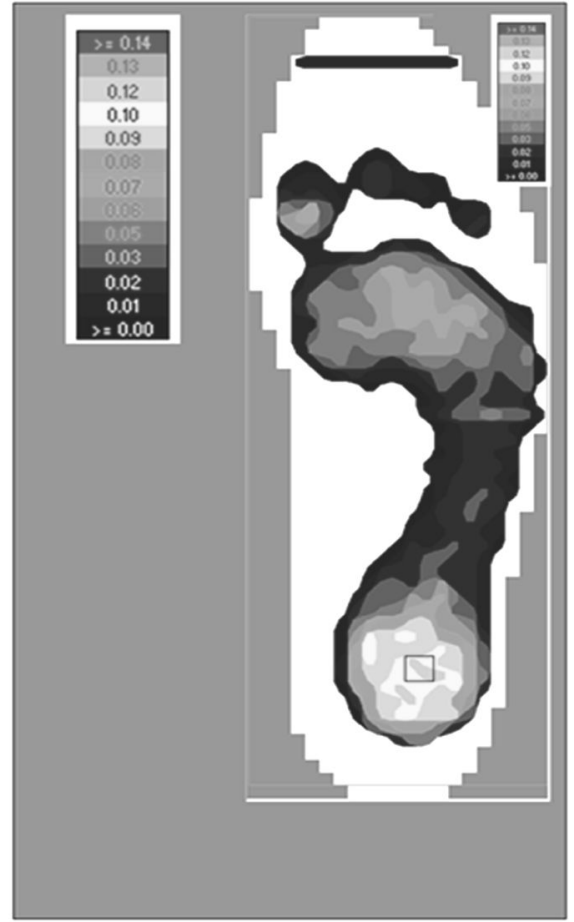

B

Figure 2. Regions interfacing with the nine region of interest $(\mathrm{RO})$ of foot plantar $(\mathrm{A})$ were compared between FE-computed plantar pressure (i.e., compressive stresses) distribution (B) and F-scan measured plantar pressure distribution (C) of the subject during standing. Both the model and the measurement showed similar patterns of plantar pressure distribution with the pressure value peaking around the center of the heel. The nine ROl in plantar soft-tissue were: the subhallucal $(H)$, the 1st submetatarsal [medial M1(M)) and lateral sesamoids $M 1(L)$ ], the 2nd to 5 th submetatarsal (M2 to M5), the lateral submidfoot (L) and the subcalcaneal (C) region. Note: FE-computed compressive stresses are denoted as negative values, with the red color indicating the region having the highest stress magnitude. 
Table 3. Comparison of FE predictions and F-scan measurements

\begin{tabular}{ccccc}
\hline & COP & PCS (MPa) & TCA & (mm2) \\
\hline \hline F-scan data & Heel & center located & 0.130 & 10,700 \\
\hline FE predictions & Heel & center located & 0.178 & 7,522 \\
\hline
\end{tabular}

The ratio (i.e, magnification factors) between the peak plantar pressure and the peak internal von Mises stress (v) were utilized to characterize the stress concentration effects that may cause a large variation of stress locally inside the plantar soft-tissue.

\section{Results}

\section{Model Validation}

As revealed in Figure 2, the FE model predicted similar patterns of plantar pressure distribution with the F-scan measures. Both the computed peak compressive stress and the measured peak plantar pressure were located beneath the center of the heel region. Forefoot at the level of metatarsal heads predicted the second highest focal stress during plantar support, followed by the big (great) toe and the midfoot which were only minimally involved in weight-bearing process. Site-to-site comparison revealed deviations of $\mathrm{FE}$ predictions from F-scan measures ranged from $11.7 \%$ to $32.5 \%$. The maximum deviation was located at M5, while M2 displayed the best match between FE prediction and F-scan data. Good agreements were also found between the FE computed foot-ground contact characteristics and F-scan data in terms of COP, PCS and TCA, especially true for the COP and PCS predictions.The internal stress beneath the adjacent foot bones was significantly higher than the external stress across all nine $\mathrm{ROI}$ of the foot plantar. The internal $\mathrm{ROI}$ generally showed over 2 folds more stress than the external. The external and internal stress at ROI M2 depicted the largest difference. The internal stress at this site was over 4.5 times greater than the external. The magnifications in other $\mathrm{ROI}$ in the descending order are: M4 with 4.4, M5 with 4.0, C with 3.5, M3 with 3.3, M1 (M\&L) with 2.0, $\mathrm{H}$ with 2.0 and $\mathrm{L}$ with 1.5. It demonstrated that stress distribution inside the plantar soft-tissue was inconsistent with the external stress distribution. For forefoot region, externally, sites M1 (M) and M1 (L) experienced the highest stress; while internally most stress was concentrated at M2, M4, and M5. The relationship between external andinternal stress of plantar soft-tissue seems to be site-dependent.

\section{Discussion}

Abnormally high foot pressures have been shown in the past to be associated with an increased risk for developing foot ulceration (Stess et al., 1997). Clinically, an empirical plantar foot pressure of $600 \mathrm{KPa}$ has been considered to be indicative of patients at high risk for foot ulceration (Delbridge et al., 1988, Pham et al., 2000). In the present study, the internal stress was found to be site-dependent and the observation showed a change between 1.5 to 4.5 times the external stress on the foot plantar surface. 
Therefore, based on the computed magnification factors, the internal stress inside the plantar soft-tissue can vary from $900 \mathrm{KPa}$ up to $2,700 \mathrm{KPa}$. Considering the local pathological stiffening(Gefen et al., 2001) or migration (displacement of fat-pad from beneath bony prominences) (Bus et al., 2004) of the plantar soft-tissue across the foot plantar, an even higher internal stress or possibly over-dosed stress can be expected inside the diabetic foot. Al-though the exact stress thresholds or the tolerance of the different tissue for normal and diabetic foot are still miss-ing, the external/internal stress associations obtained from the current foot model can serve as a useful baseline case for future work.

\section{References}

Alexander, I. J., Chao, E. Y., \& Johnson, K. A.(1990). The assessment of dynamic foot-to-ground contact forces and plantar pressure distribution: a review of the evolution of current techniques and clinical applications. Foot \& Ankle International, 11, 152-67.

Armstrong, D. G., Peters, E. J., Athanasiou, K. A., \& Lavery, L. A.(1998). Is there a critical level of plantar foot pressure to identify patients at risk for neuropathic foot ulceration? Joumal Foot Ankle Surgery, 37, 303-7.

Athanasiou, K. A., Liu, G. T., Lavery, L. A., Lanctot, D. R., \& Schenck, R. C., Jr.(1998.) Biomechanical topography of human articular cartilage in the first metatarsophalangeal joint. Clinical Orthopaedic Related Research, 269-81.

Boulton, A. J., Vileikyte, L., Ragnarson-Tennvall, G., \& Apelqvist, J.(2005). The global burden of diabetic foot disease. Lancet, 366, 1719-24.
Brash, P. D., Foster, J. E., Vennart, W., Daw, J., \& Tooke, J. E.(1996). Magnetic resonance imaging reveals micro-haemorrhage in the feet of diabetic patients with a history of ulceration. Diabetic Medicine, 13, 973-8.

Bus, S. A., Maas, M., Cavanagh, P. R., Michels, R. P., \& Levi, M.(2004). Plantar fat-pad displacement in neuropathic diabetic patients with toe deformity: a magnetic resonance imaging study. Diabetes Care, 27, 2376-81.

Bygrave, C. J., \& Betts, R. P.(1992). The plantar tissue thickness in the foot: a new ultrasound technique for loadbearing measurements and a metatarsal head depth study. The Foot, 3, $71-8$.

Cavanagh, P. R., Ulbrecht, J. S., \& Caputo, G. M.(2000). New developments in the biomechanics of the diabetic foot. Diabetes Metabolism Research Reviews, 16 Suppl 1, S6-S10.

Delbridge, L., Perry, P., Marr, S., Arnold, N., Yue, D. K., Turtle, J. R., \& Reeve, T. S.(1988). Limited joint mobility in the diabetic foot: relationship to neuropathic ulceration. Diabetic Medicine, 5, 333-7.

Duckworth, T., Boulton, A. J., Betts, R. P., Franks, C. I., \& Ward, J. D.(1985). Plantar pressure measurements and the prevention of ulceration in the diabetic foot. Journal Bone Joint Surgery British, 67, 79-85.

Gefen, A.(2003). Plantar soft tissue loading under the medial metatarsals in the standing diabetic foot. Medicine Engineer Physical, 25, 491-9.

Gefen, A., Megido-Ravid, M., Azariah, M., Itzchak, Y., \& Arcan, M.(2001). Integration of plantar soft tissue stiffness measurements in routine MRI of the diabetic foot. Clinical Biomechanics, (Bristol, Avon) 16, 921-5.

Keyak, J. H., Rossi, S. A., Jones, K. A., \& Skinner, H. 
B.(1998). Prediction of femoral fracture load using automated finite element modeling. Journal of Biomechanics, 31, 125-33.

Lemmon, D., Shiang, T. Y., Hashmi, A., Ulbrecht, J. S., \& Cavanagh, P. R.(1997). The effect of insoles in therapeutic footwear a finite element approach. Joumal of Biomechanics, 30, 615-20.

Masson, E. A., Hay, E. M., Stockley, I., Veves, A., Betts, R. P., \& Boulton, A. J.(1989). Abnormal foot pressures alone may not cause ulceration. Diabetic Medicine, 6, 426-8.

Morag, E., \& Cavanagh, P. R.(1999). Structural and functional predictors of regional peak pressures under the foot during walking. Joumal of Biomechanics, 32, 359-70.

Mueller, M. J., Hastings, M., Commean, P. K., Smith, K. E., Pilgram, T. K., Robertson, D., \& Johnson, J.(2003). Forefoot structural predictors of plantar pressures during walking in people with diabetes and peripheral neuropathy. Joumal of Biomechanics, 36, 1009-17.

Nakamura, S., Crowninshield, R. D., \& Cooper, R. R.(1981). An analysis of soft tissue loading in the foot a preliminary report. Bulletin of Prosthetics Research, 18(1), 27-34.

Pham, H., Armstrong, D. G., Harvey, C., Harkless, L. B., Giurini, J. M., \& Veves, A.(2000. Screening techniques to identify people at high risk for diabetic foot ulceration: a prospective multicenter trial. Diabetes Care, $23,606-11$.

Siegler, S., Block, J., \& Schneck, C. D.(1988). The mechanical characteristics of the collateral ligaments of the human ankle joint. Foot \& Ankle International, 8, 234-42.

Singh, N., Armstrong, D. G., \& Lipsky, B. A.(2005). Preventing foot ulcers in patients with diabetes. Joumal of American Medical Association, 293, 217-28.

Stess, R. M., Jensen, S. R., \& Mirmiran, R.(1997). The role of dynamic plantar pressures in diabetic foot ulcers. Diabetes Care, 20, 855-8.

Wright, D. G., \& Rennels, D. C.(1964). A Study of the Elastic Properties of Plantar Fascia. Journal of Bone Joint Surgery American, 46, 482-92.

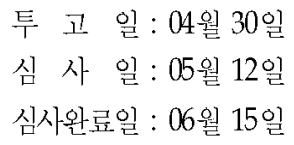

